\title{
Cardiomiopatía de Takotsubo, a más de 30 ańos de su descripción: Reporte de caso y Revisión de la literatura
}

\author{
Takotsubo Cardiomyopathy - More than 30 years after \\ its description. Case report and literature review
}

Nasly Violedy Ipia Ordónez ${ }^{1}$, Miller Giraldo Sandoval ${ }^{2}$

\section{RESUMEN}

La Cardiomiopatía de Takotsubo es una enfermedad cardíaca aguda con una presentación clínica similar al sindrome coronario agudo. Afecta principalmente a mujeres posmenopáusicas, a menudo precedido por estrés emocional o físico. Pacientes con factores predisponentes como malignidad y comorbilidades crónicas son más propensas a sufrir este síndrome.

\section{ABSTRACT}

Takotsubo Cardiomyopathy is an acute heart disease with a clinical presentation similar to acute coronary syndrome. It mainly affects postmenopausal women, often preceded by emotional or physical stress. Patients with predisposing factors such as malignancy and chronic comorbidities are more likely to suffer from this syndrome. The pathogenesis is unclear and have

\section{Historial del artículo:}

Fecha de recepción: 21/02/2019

Fecha de aprobación: 15/06/2019

1 Clínica La Estancia. Médico General. Unidad de Angiografía. Popayán, Colombia.

2 Hospital Universitario del Valle. Clínica Miocardio. Médico Especialista en Medicina Interna, Cardiología, Hemodinamia y Cardiología Intervencionista. Cali, Valle. Colombia.

Correspondencia: Calle 73 AN, 4 B 46, Villa del Norte. Popayán, Colombia. Teléfono: 312-7729301. Correo electrónico: nasly_ipia@ hotmail.com

Como citar este artículo: Ipia-Ordónez N, Giraldo-Sandoval M. Cardiomiopatía de Takotsubo, a más de 30 ańos de su descripción: Reporte de caso y Revisión de la literatura. Revista de la Facultad de Ciencias de la Salud de la Universidad del Cauca. 2019; 21(2): 32 - 37 
La patogénesis no está clara y se han propuesto como mecanismos: espasmo coronario, disfunción microvascular, infarto miocárdico abortado, obstrucción del tracto de salida del ventrículo izquierdo, toxicidad miocárdica por catecolaminas, y disfunción autonómica; pero hasta el momento no se ha logrado establecer con claridad la causa exacta de esta patología. Se describe el caso de una mujer posmenopáusica con un cuadro clínico inicial similar a un Síndrome Coronario Agudo, quien fue llevada a coronariografia como parte del protocolo del Síndrome Coronario, sin lesiones significativas, pero en la ventriculografía se obserú el abalonamiento apical, característico de este síndrome. Se inició manejo de soporte, alcanzando la recuperación completa de la paciente, no obstante, hasta el momento no se ha descrito un manejo específico para esta patología. Se requieren más investigaciones para aclarar la fisiopatología de esta Cardiomiopatía y así protocolizar un manejo de terapia dirigida que logre un mejor pronóstico en nuestros pacientes.

Palabras clave: Cardiomiopatía de Takotsubo, / fisiopatología, Diagnóstico, Tratamiento de Urgencia, Informes de Casos. (DeCS)

\section{INTRODUCCIÓN}

La Cardiomiopatía de Takotsubo (TS) es un síndrome caracterizado por signos y síntomas similares al infarto agudo de miocardio en ausencia de dańo en las arterias coronarias. Es más común en mujeres postmenopáusicas, solo unos pocos casos han sido reportados en mujeres jóvenes y es muy raro en mujeres embarazadas (1). Antes de la introducción del término takotsubo, el síndrome existió bajo diferentes diagnósticos. Desde la introducción del término japonés takotsubo en 1990, el síndrome ha sido cada vez más reconocido en casi todos los países de los seis continentes del mundo. TS ha sido informado en una variedad de razas, pero de acuerdo con algunos informes, es poco común en hispanos y afroamericanos. Se ha informado que la prevalencia mundial de TS es aproximadamente $2 \%$ (hasta $10 \%$ si solo se consideran mujeres) de todos los pacientes que se presentan con manifestaciones clínicas de Síndrome Coronario Agudo (2). La prevalencia está subestimada y la razón principal de esto es el desconocimiento de la enfermedad. Sin embargo, con una mayor conciencia y un acceso más generalizado a la angiografía coronaria invasiva temprana, TS es ahora reconocido con mayor frecuencia. been proposed as mechanisms: coronary spasm, microvascular dysfunction, aborted myocardial infarction, obstruction of the left ventricular outflow tract, myocardial toxicity due to catecholamines, and autonomic dysfunction. But until now it has not been possible to establish with clarity the exact cause of this pathology. The case of a postmenopausal woman with initial diagnosis of Acute Coronary Syndrome is described. In the coronary angiography, no significant lesions were found, and in the ventriculography the apical abalonation was observed, which is characteristic of this syndrome. In this case there was a complete recovery of the patient. More research is needed to try to elucidate clearly the pathophysiology of this syndrome and thus, try to protocolize a management to have a targeted therapy that achieves a faster recovery, without sequelae and a better prognosis in our patients.

Keywords:Takotsubo Cardiomyopathy, /physiopathology, Diagnosis, Emergency Treatment, Case reports. (MeSH)

Se describe como una cardiomiopatía primaria. Según la clasificación de la Asociación Americana del Corazón, el Síndrome de Takotsubo incluye la miocardiopatía de takotsubo, cardiomiopatía por estrés, abalonamiento apical, y "corazón roto". Un takotsubo es una trampa japonesa de pulpo ampliamente usada alrededor del canal de Akashi en la prefectura de Hyogo y el mar interior de Seto desde hace varios siglos (Figura 1). En 1986, el New England Journal of Medicine presentó un reporte de caso de una mujer de 44 ańos que sufrió de dolor torácico después de un estrés emocional severo, se presume que es el primer caso reportado de Síndrome de Takotsubo fuera de Japón. En 1977, el Dr. Kuramoto informó 7 casos de infarto agudo de miocardio reversible después de una transfusión sanguínea en pacientes ancianos, aunque su estudio no presentó hallazgos de ventriculogramas izquierdos o ecocardiogramas, el curso de la evolución registrado por electrocardiografía fue bastante similar al de los pacientes con Síndrome de Takotsubo (3). En 1990, el término takotsubo fue descrito en la literatura médica por el Dr. Hikaru Sato, del hospital de la ciudad de Hiroshima en Hiroshima, Japón. El título de este informe era "tsubo" miocardio aturdido escrito en japonés, en inglés el título fue renombrado como "tako-tsubo, como la disfunción del 
ventrículo izquierdo". En 1991, el Dr. Dote, uno de los colegas del Dr. Sato, presentó un estudio titulado "El aturdimiento del miocardio debido a un espasmo coronario multivaso simultáneo" que fue la primera serie de casos takotsubo publicada en una revista revisada por pares en japonés con un resumen en inglés. El informe de este estudio fue citado con frecuencia por numerosas revistas científicas debido a las impresionantes cifras, incluyendo 5 casos representados mediante el uso de ventriculografía izquierda (3).

En el presente artículo se reporta el caso de una paciente de 52 ańos de edad que se presentó al servicio de urgencias con signos y síntomas compatibles con un síndrome coronario agudo sin evidencia angiográfica de enfermedad coronaria, en quien se diagnosticó por ventriculografía y ecocardiografía un Síndrome de Takotsubo.

\section{DESCRIPCIÓN DEL CASO}

Paciente de género femenino, de 52 ańos de edad con antecedente de hipertensión arterial y obesidad, sin otros antecedentes patológicos conocidos; de ocupación ama de casa, procedente del municipio de Caldono, departamento del Cauca. Quien ingresó al servicio de urgencias de Clínica La Estancia de la Ciudad de Popayán (Cauca - Colombia), el 4/10/2017, por cuadro clínico de 3 horas de evolución de dolor torácico anginoso asociado a disnea, progresando rápidamente a falla ventilatoria por edema pulmonar agudo y choque cardiogénico, con requerimiento de soporte ventilatorio invasivo, manejo con diurético endovenoso, antiagregantes plaquetarios y heparina de bajo peso molecular. Troponina inicial y de control positivas. Electrocardiograma sugestivo de

Figura 1. Recipiente japonés para pulpos, llamado "Takotsubo"

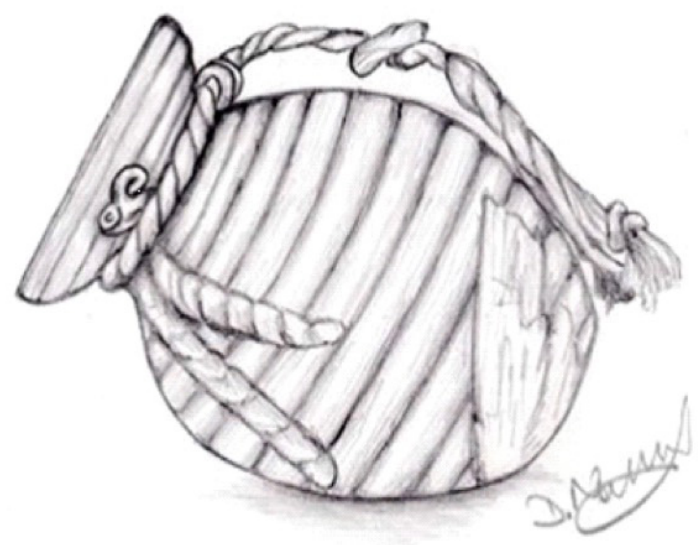

isquemia anteroseptal (Figura 2). Ecocardiograma inicial con FEVI de 35-40\%, hipocinesia de todos los segmentos medios y del casquete apical, solo se contraían de forma adecuada los segmentos basales. Arteriografía coronaria: coronarias epicardicas sin lesiones; ventriculograma izquierdo: hipoquinesia moderada anterolateral, apical e infradiafragmática, FEVI de 35-40\% (Figura 3). Inician soporte vasoactivo con norepinefrina, y soporte inotrópico con levosimendan durante 48 horas con retiro posterior sin complicaciones, extubada igualmente sin complicaciones 24 horas después del inicio de la ventilación mecánica. Estando en el área de hospitalización 4 días después de su ingreso se documenta bradicardia sin colapso hemodinámico, Holter EKG 24 horas con bloqueo AV II grado - Mobitz II, el cual se considera crónico por no asociar síntomas. Ecocardiograma de control con FEVI de 45-50\%, hipocinesia global leve, sin defectos segmentarios. Valorada por psiquiatría quien hace diagnóstico de síndrome de ansiedad e inicia manejo con ansiolítico. Es egresada el 29 de octubre de 2017 con manejo anti falla (Carvedilol, espironolactona, hidroclorotiazida, losartan, atorvastatina, ASA) y ansiolítico (alprazolam). Control ambulatorio con Medicina Interna el 13/12/17 don encuentran con cifras de presión arterial elevada, y síntomas ansiosos marcados; ajustan manejo antihipertensivo y derivan de forma inmediata a consulta por psiquiatría.

\section{DISCUSIÓN}

La Cardiomiopatía de Takotsubo es una entidad recientemente reconocida como una enfermedad cardíaca aguda con presentación clínica similar al síndrome coronario agudo. Lo típico en estos pacientes es que adquiere un patrón circunferencial izquierdo con anormalidad en la contracción

Figura 2. Electrocardiograma inicial: ondas T hiperagudas en cara anteroseptal.

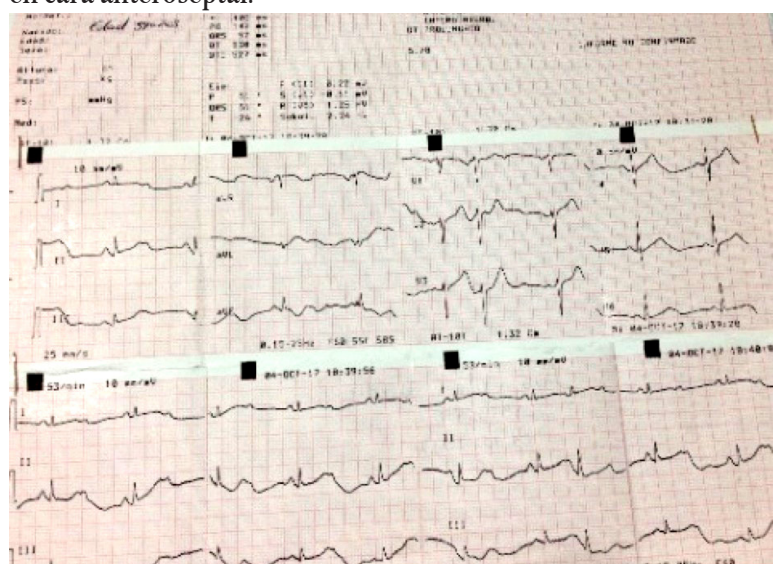


ventricular que se extiende más allá de un territorio de suministro de una arteria coronaria y parece sigue la inervación simpática cardiaca anatómica, como se describe en nuestra paciente en quien se evidenció hipocinesia de todos los segmentos medios y del casquete apical en ecocardiograma e hipoquinesia moderada anterolateral, apical e infradiafragmática en el ventriculograma izquierdo comprometiendo la arteria descendente anterior y sus ramas. El síndrome afecta predominantemente a mujeres posmenopáusicas y a menudo está precedido por un estrés emocional o físico (4). Pacientes con factores predisponentes como malignidad y otras comorbilidades crónicas son más propensas a sufrir de TS. La patogénesis del síndrome no está bien dilucidada, varios mecanismos fisiopatológicos que involucran isquemia miocárdica (espasmo multivaso de arterias coronarias, disfunción microvascular, infarto de miocardio abortado), obstrucción del tracto de salida del ventrículo izquierdo, toxicidad miocárdica mediada por catecolaminas, cambiosinducidos por epinefrina en el tráfico de seńales y disfunción del sistema nervioso autónomo han sido propuestos $(5,6)$. A continuación, se discuten con más detalles los avances hechos en el entendimiento de la fisiopatología, con el fin de tener mayor claridad sobre lo que sucede en el miocardio para poder llegar a plantear un manejo más específico en futuros pacientes.

Cuando Sato y Dote introdujeron el término Takotsubo consideraron que la disfunción ventricular izquierda era secundaria a vasoespasmo coronario múltiple, esto se basó en el hecho de que ellos demostraron vasoespasmo en la angiografía coronaria de dos pacientes y observaron espasmo en otros dos pacientes después de la administración de ergonovina. En series posteriores, la prevalencia de vasoespasmo coronario como causa de isquemia miocárdica ha sido variable, se ha informado solamente en 5-10\%, lo que implica que la mayoría de los pacientes no tenían vasoespasmo coronario espontáneo (7). El vasoespasmo inducido ha sido informado en un número limitado de pacientes en la fase aguda de TS. En una revisión de nueve estudios, las pruebas de provocación podrían inducir vasoespasmo coronario en solo 34 de 123 pacientes (28\%) (7). La coronariografía de la paciente tomada en la fase aguda del evento no demostró ni lesiones obstructivas ni presencia de espasmo coronario concordante con lo reportado en la literatura.

La disfunción microvascular ha sido defendida por varios investigadores como un posible mecanismo fisiopatológico del movimiento anormal del ventrículo izquierdo en TS. La angiografía coronaria revela arterias coronarias normales en la mayoría de los pacientes, pero una limitación importante de la angiografía coronaria es su incapacidad para visualizar la microcirculación coronaria. Estudios que usan técnicas invasivas semicuantitativas como recuento de flujo sanguíneo coronario (TIMI) en infarto de miocardio, flujo TIMI corregido, grado de perfusión miocárdica y reserva de flujo coronario, han mostrado resultados conflictivos, al evaluar el flujo TIMI, Fazio et al. Informaron flujo coronario lento en 23 de 24 pacientes con TS durante la etapa aguda.

Figura 3. A: Ventriculograma izquierdo normal. B: Ventriculograma izquierdo de la paciente: hipoquinesia anterolateral, apical e infradiafragmatica que dan la imagen similar a un "takotsubo".

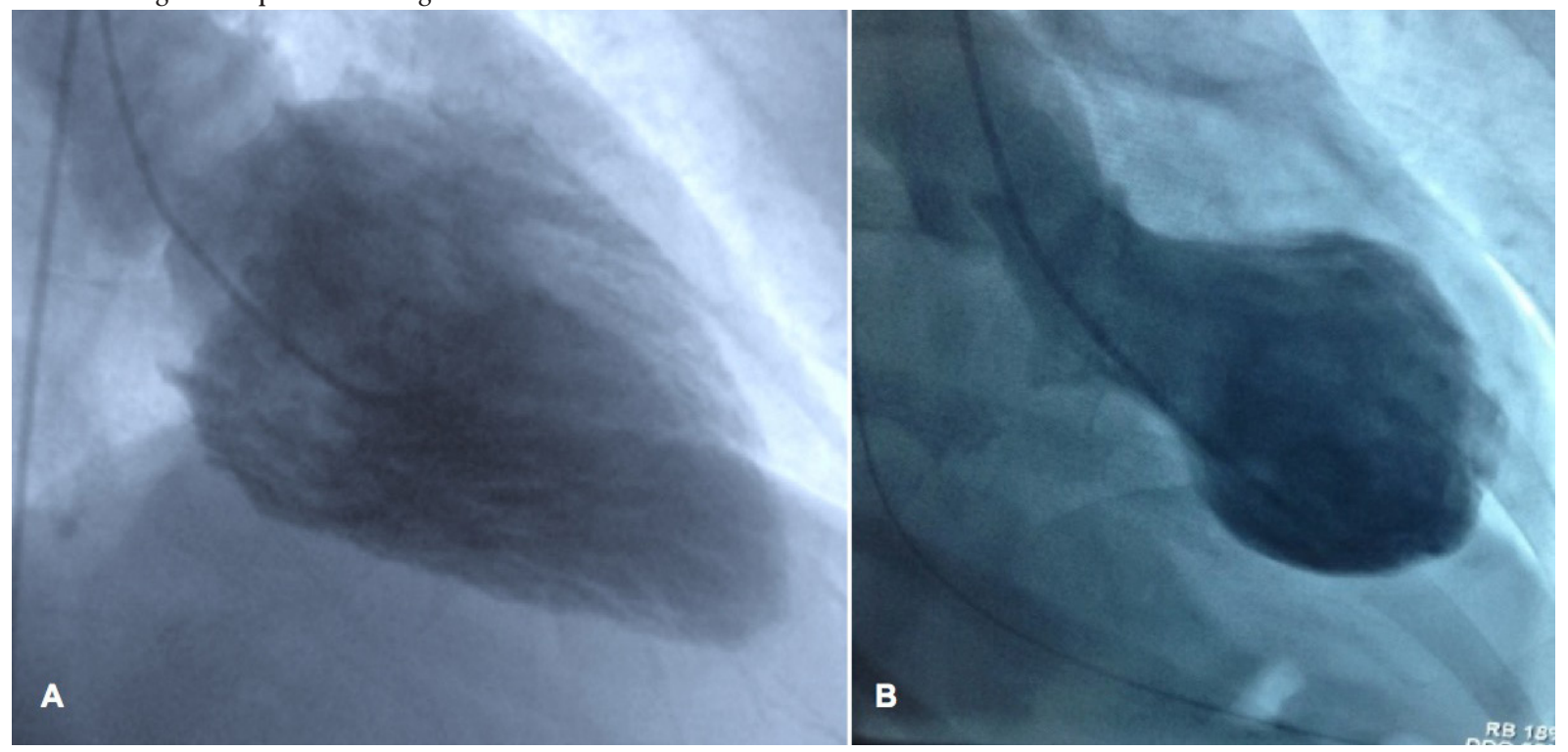


En otro estudio, Sharkey et al. No encontraron diferencias significativas en mediciones de conteo de flujo TIMI en 59 pacientes con TS en comparación con los controles (2).

La toxicidad del miocardio mediada por catecolaminas ha sido sugerida como uno de los mecanismos fisiopatológicos del TS. Esto se basa en la historia de estrés emocional anterior al inicio de la enfermedad en muchos pacientes, el informe de niveles extremadamente altos de catecolaminas en plasma en pacientes con TS; la ocurrencia de TS en pacientes con feocromocitoma; y la inducción de TS por administración terapéutica y accidental de epinefrina, norepinefrina y otros inotrópicos de catecolaminas apoyan esta hipótesis (8). Consideramos que esta puede ser la base de la disfunción cardíaca aguda de nuestra paciente teniendo en cuenta la valoración psiquiátrica que se hace de ella donde se diagnostica un síndrome de ansiedad crónico, el cual en control ambulatorio aún no se ha logrado estabilizar.

Varios investigadores han proporcionado pruebas sustanciales de la participación del sistema nervioso simpático, incluidos hiperactivación e interrupción del sistema nervioso simpático cardíaco y los niveles de norepinefrina en la fisiopatología de TS. La ocurrencia del síndrome después de eventos emocionales desencadenantes, el aturdimiento del miocardio con un patrón circunferencial peculiar, que puede seguir la distribución local del nervio simpático cardíaco, los hallazgos histopatológicos de necrosis y la evidencia de miocarditis con edema de miocardio se encuentran entre los datos de apoyo para la implicación del sistema nervioso simpático cardíaco local en la fisiopatología de TS (9).

A pesar de los avances en el entendimiento de la fisiopatología, los estudios actuales no muestran una evidencia fuerte a favor de cada una de las teorías propuestas, lo cual hace necesario continuar realizando estudios que nos permitan dilucidar los mecanismos subyacentes del TS para que en un futuro nuestros pacientes reciban tratamientos específicos lo cual aseguraría su recuperación en un tiempo menor.

El tratamiento para los casos leves con mejoría sintomática rápida no es específico, consiste en el manejo sintomático, y es posible el alta temprana. Algunos autores abogan por terapia convencional de falla cardíaca con introducción gradual de inhibidores de la enzima convertidora de angiotensina (IECA) y beta bloqueadores. Sin embargo, la evidencia de que una proporción sustancial de pacientes con TS puede tener alteración en el sistema simpático periférico asociada a baja resistencia vascular periférica, indicaría que la administración de inhibidores de la ECA, betabloqueadores, bloqueadores de los canales de calcio u otras drogas vasoactivas pueden ser dańinas. Por tanto, la seguridad y eficacia de estos agentes farmacológicos en el tratamiento de pacientes con TS aún no sido establecido (10). En nuestro caso el manejo con vasoactivo e inotrópico demostró eficacia notable, con una mejoría rápida de la función ventricular documentada en el ecocardiograma de control, este manejo si bien ofreció beneficios para la paciente aún no está establecido en guías médicas como protocolo de manejo para pacientes con TS. El tratamiento de los factores precipitantes, enfermedades predisponentes y complicaciones es fundamental durante la etapa aguda de la enfermedad. Nuestra paciente además de recibir soporte ventilatorio, inotrópico y vasoactivo, también recibió terapia antifalla convencional con una buena respuesta en la recuperación de la FEVI y terapia ansiolítica guiada por psiquiatría.

En cuanto al pronóstico en pacientes con TS, el abalonamiento apical típico está asociado con una disfunción más severa del ventrículo izquierdo en la presentación aguda y una tasa de mortalidad más alta dentro de los primeros 6 meses después del evento inicial. Después de la recuperación completa de la función del ventrículo izquierdo, el pronóstico es similar en pacientes con patrones de abalonamientos típicos y atípicos (11). El TS se asocia con un deterioro agudo de las variables convencionales de la función diastólica la cual mejora durante la recuperación y puede ocurrir en paralelo con la recuperación de la función sistólica (12). El síndrome generalmente es reversible; sin embargo, durante la fase aguda, un número considerable de los pacientes desarrollan complicaciones graves, como arritmias, insuficiencia cardíaca, edema agudo de pulmón y choque cardiogénico; tromboembolismo, paro cardíaco y ruptura de pared libre. En este caso la paciente no tuvo complicaciones asociadas al síndrome lo cual mejora notablemente su pronóstico a largo plazo.

\section{CONCLUSIÓN}

La Cardiomiopatía de Takotsubo es un síndrome poco frecuente que se puede confundir con otras condiciones que tienen signos y síntomas similares, como el infarto agudo de miocardio, tromboembolismo pulmonar o miocardiopatía dilatada aguda; por lo tanto, es importante realizar un diagnóstico diferencial adecuado porque cada condición tiene un tratamiento y un pronóstico diferentes. Una vez que los pacientes son diagnosticados correctamente, su tratamiento es de soporte, guiado según la condición clínica individual. La mayoría tiene una recuperación completa dentro de los tres a cuatro meses 
siguientes al evento. Se requieren más investigaciones que intenten dilucidar con mayor claridad la fisiopatología de este síndrome y así intentar protocolizar una terapia dirigida que logre un mejor pronóstico en los pacientes.

\section{DECLARACIÓN DE CONFLICTOS DE INTERÉS}

Los autores declaran no tener conflictos de interés.

\section{AGRADECIMIENTOS}

Angiografía de Occidente, Unidad de hemodinámia, Popayán - Cauca, Colombia. Sitio donde se realizó el cateterismo cardíaco.

\section{REFERENCIAS}

1. Ruiz S, Martinez-Marin M, Luque P, Nassar N, Oros D. Takotsubo cardiomyopathy after cesarean section: A case report and literature review. J Obstet Gynaecol Res. 2017; 43(2):392-396.

2. Y-Hassan S, Tornvall P. Epidemiology, pathogenesis, and management of takotsubo syndrome. Clin Auton Res. 2018; 28(1):53-65.

3. Akashi, Yoshihiro J., Masaharu I. Takotsubo Syndrome. Insights from Japan. Heart Failure Clinics. 2016; 12:587-595.

4. Illera D, Rivera G, Orozco A, Montenegro V, Vidal C. Perfil epidemiológico y factores de riesgo en pacientes de la unidad de cuidados intensivos, Hospital San José, Popayán. Revista Facultad Ciencias de la Salud. Universidad del Cauca. 2015; 17(1): 14-19.

5. Shoemaker R. La inferencia causal. Revista Facultad Ciencias de la Salud. Universidad del Cauca. 2014; 16(1): 44-47.

6. Ghadri JR, Cammann VL, Templin C. The International Takotsubo Registry Rationale, Design, Objectives, and First Results. Heart Fail Clin. 2016; 12(4):597-603.

7. Akashi YS, Nef HM, Lyon AR. Epidemiology and pathophysiology of Takotsubo syndrome. Nat Rev Cardiol. 2015; 12(7):387-97.

8. Sharkey SW, Windenburg DC, Lesser JR, Maron MS, Houser RG, LesserJN, et al. Natural History and Expansive Clinical Profile of Stress (Tako-Tsubo) Cardiomyopathy. J Am Coll Cardiol. 2010; 55 (4) 333-341.

9. Y-Hassan S. The pathogenesis of reversible T-wave inversions or large upright peaked T-waves: Sympathetic T-waves. Int J Cardiol. 2015; 191:237-43.
10. Omerovic E. Takotsubo Syndrome - Scientific Basis for Current Treatment Strategies. Heart Failure Clinics. 2016; 12(4): 577-586.

11. Stiermaier T, Möller C, Graf T, Eitel C, Desch S, Thiele H, Eitel I. Prognostic Usefulness of the Ballooning Pattern in Patients With Takotsubo Cardiomyopathy. Am J Cardiol. 2016; 118(11):1737-1741.

12. Kumar S, Waldenborg M, Bhumireddy P, Ramkissoon $\mathrm{K}$, Loiske K, et al. Diastolic function improves after resolution of takotsubo cardiomyopathy. Clin Physiol Funct Imaging. 2016; 36(1):17-24. 